\title{
Method for holographic storage using peristrophic multiplexing
}

\author{
Kevin Curtis, Allen Pu, and Demetri Psaltis \\ Department of Electrical Engineering, California Institute of Technology, MS 116-81, Pasadena, California 91125 \\ Received January 10, 1994

\begin{abstract}
A method of multiplexing holograms by rotating the material or, equivalently, the recording beams is described. Peristropic (Greek for rotation) multiplexing can be combined with other multiplexing methods to increase the storage density of holographic storage systems. Peristrophic multiplexing is experimentally demonstrated with Du Pont's HRF-150 photopolymer film. We multiplexed a total of 295 holograms in a 38- $\mu$ m-thick photopolymer film by combining peristrophic multiplexing with angle multiplexing.
\end{abstract}

The number of holograms that can be multiplexed in a given holographic system is primarily a function of two parameters - the system's bandwidth (either temporal or spatial frequency) and the material's dynamic range. Recently, thin-film materials have been developed with relatively large dynamic range. An example of such a material is Du Pont's HRF-150 photopolymer. ${ }^{1}$ Previously we reported 10 angle-multiplexed holograms in a $38-\mu \mathrm{m}$-thick film ${ }^{2}$ with a diffraction efficiency of $10^{-3}$. Since typically we can work with holographic diffraction efficiencies of the order of $10^{-6}$, we have sufficient dynamic range to record sufficiently more than $\mathbf{1 0}$ holograms. The angular bandwidth limitation can be alleviated by making the film thicker, ${ }^{3}$ but scattering increases rapidly with thickness in these materials. Another method that has been used to increase the utilization of the available bandwidth of the system is fractal sampling grids. ${ }^{4,5}$

In this Letter we describe peristrophic (Greek for rotation) multiplexing as a solution to the bandwidthlimited capacity problem. With this method the hologram is physically rotated, with the axis of rotation being perpendicular to the film's surface every time a new hologram is stored. The rotation does two things. It shifts the reconstructed image away from the detector, permitting a new hologram to be stored and viewed without interference, and it can also cause the stored hologram to become non-Bragg matched. In addition, peristrophic multiplexing can be combined with other multiplexing techniques such as angle and wavelength multiplexing to increase the storage density and with spatial multiplexing to increase the storage capacity of the system.

The setup for peristrophic multiplexing Fourier plane holograms is shown in Fig. 1. The reference plane wave $(R)$ is incident at an angle $\theta_{r}$, and the signal beam $(S)$ is incident at an angle $\theta_{s}$, both angles measured with respect to the film's normal. Taking the center pixel of the image as the signal and neglecting any effects due to hologram thickness, we can write the hologram transmittance as

$$
R^{*} S=\exp \left(-i 2 \pi \frac{\sin \theta_{r}}{\lambda} x\right) \exp \left(-i 2 \pi \frac{\sin \theta_{s}}{\lambda} x\right) .
$$

The hologram is then rotated by $\mathrm{d} \theta$ about the center of the $x-y$ plane, as shown in Fig. 1 . If it is assumed that the rotation is small, this results in the coordinates' being transformed according to $x^{\prime} \approx x-y \mathrm{~d} \theta$ and $y^{\prime} \approx y+x \mathrm{~d} \theta$. Substituting these relations into Eq. (1), we can express the hologram in terms of the unrotated coordinates $(x, y)$ :

$$
\begin{aligned}
R^{*} S= & \exp \left(-i \frac{2 \pi \sin \theta_{r} x}{\lambda} x\right) \exp \left(-i \frac{2 \pi \sin \theta_{s} x}{\lambda}\right) \\
& \times \exp \left[-i \frac{2 \pi\left(\sin \theta_{s}+\sin \theta_{r}\right) \mathrm{d} \theta y}{\lambda}\right] .
\end{aligned}
$$

After multiplication by $R$ and Fourier transforming, the last term in Eq. (2) results in a shift in the image. The rotation required for translation of the image out of the detector aperture is approximately given by

$$
\mathrm{d} \theta \geq \frac{d / F}{\sin \theta_{s}+\sin \theta_{r}},
$$

where $d$ is the size of the image detector plane and $F$ is the focal length of the lens used. For image-plane holograms the expression is ${ }^{6}$

$$
\mathrm{d} \theta \geq \frac{2 \lambda / \delta}{\sin \theta_{s}+\sin \theta_{r}},
$$

where $1 / \delta$ is the highest spatial frequency in the image. For image-plane holograms, the undesired holograms are filtered out at the Fourier plane of the system. Notice that this method can be combined with other volumetric multiplexing methods to increase the storage density further.

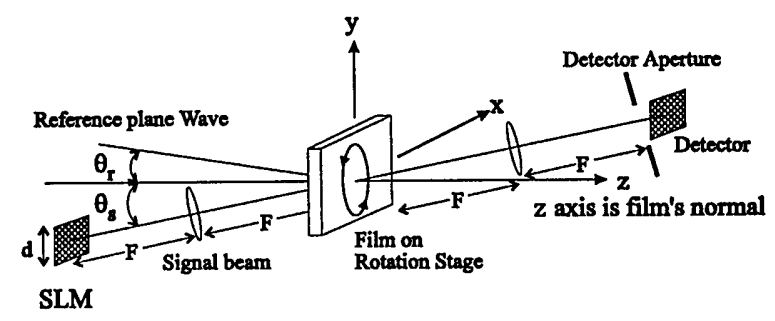

Fig. 1. Setup for peristrophic multiplexing. SLM, spatial light modulator. 


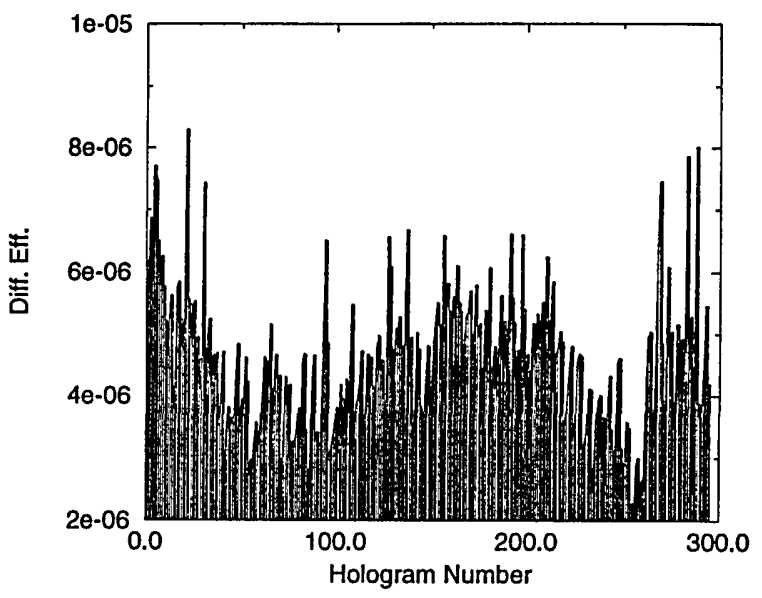

Fig. 2. Diffraction efficiency versus hologram number for 295 holograms stored in $38-\mu \mathrm{m}$-thick film.

The Bragg selectivity, assuming that the reference is given by

$$
R=\exp \left[-i\left(\frac{2 \pi \sin \theta_{r}}{\lambda} x+\frac{2 \pi \cos \theta_{r}}{\lambda} z\right)\right]
$$

and the signal is given by

$$
S=\exp \left[-i\left(\frac{2 \pi \sin \theta_{r}}{\lambda} x+\frac{2 \pi \cos \theta_{r}}{\lambda} z\right)\right],
$$

can be calculated with the Born and the paraxial approximations and integration over the volume of the hologram. Assuming that the tranverse $(x, y)$ dimensions of the film are much larger than the bandwidth of the images, we can show the Bragg selectivity to be

$$
\mathrm{d} \theta=\left\{\frac{2 \lambda}{t}\left[\frac{\cos \theta_{s}}{\sin \theta_{r}\left(\sin \theta_{s}+\sin \theta_{r}\right)}\right]\right\}^{1 / 2},
$$

where $t$ is the thickness of the material. Using $\lambda=$ $488 \mathrm{~nm}, t=38 \mu \mathrm{m}$, and $\theta_{s}=\theta_{r}=30^{\circ}$ results in a selectivity of approximately $12^{\circ}$. The Braggmatching requirement is the dominant effect if $d / F>$ $\left[2 \lambda \cos \theta_{s}\left(\sin \theta_{s}+\sin \theta_{r}\right) / t \sin \theta_{r}\right]^{1 / 2}$. For most material thicknesses, the Bragg-matching criterion determines the required rotation for peristrophic multiplexing. In our experiments, because the thickness of the film is only $38 \mu \mathrm{m}$, the image could be filtered out before the gratings become non-Bragg matched.

The experimental setup is the same as in Fig. 1, except that a rotation stage was added to rotate the film around a vertical axis as well as around the film's normal. This makes it possible to combine peristrophic and angle multiplexing. The film was located a significant distance from the Fourier plane so that the signal beam was approximately uniform. For each peristrophic position, multiple holograms are stored by standard angle multiplexing by rotation of the medium. A spatial light modulator was used to present images (cartoons) to the system. Each frame is numbered according to the sequence in which it was stored. The reference and signal beams were initially incident at $\pm 30^{\circ}$ from the film's normal. The reference-beam intensity was $1.1 \mathrm{~mW} / \mathrm{cm}^{2}$, and the signal-beam power was $300 \mu \mathrm{W}$ in an approximately $1 \mathrm{~cm} \times 0.5 \mathrm{~cm}$ area. The film was rotated in plane by $3^{\circ}$ between each set of anglemultiplexed holograms to permit the other holograms to be filtered out. Relation (3) predicts a required rotation of approximately $9^{\circ}$ for the Fourier-plane hologram, while relation (4) predicts an approximately $1.7^{\circ}$ rotation for the image plane. The $3^{\circ}$ was experimentally observed for the in-between (Fresnel) case that we used. Each angle-multiplexed hologram was also separated by $3^{\circ}$. The initial exposure time was $0.11 \mathrm{~s}$, but, starting at hologram number 26, we exposed each hologram for 0.005 longer than the previous hologram to correct for the lost sensitivity because of run time. ${ }^{2}$ There was a 1.5-s delay between holograms to allow the rotation stages to stop completely. 295 holograms were stored in the polymer by peristrophic multiplexing 59 times and storing 5 angle-multiplexed holograms with each peristrophic position. The diffraction efficiency of the 295 holograms is plotted in Fig. 2. The average efficiency was $\sim 4 \times 10^{-6}$, and the variations are due primarily to variation in the average intensity of the frames. In a separate experiment, we stored equal-amplitude plane-wave holograms and observed a decrease in diffraction efficiency proportional to $1 / M^{2}$ ?

Previously we stored $M=10$ holograms with a roughly $10^{-13}$ diffraction efficiency, ${ }^{2}$ limited by the angular bandwidth of the optical system. Peristrophic multiplexing made it possible to store $M=295$ holograms at the same location with a diffraction efficiency of $\sim 4 \times 10^{-6}$. Thus, peristrophic multiplexing permitted an almost 2-orders-of-magnitude increase in the storage capacity of the Du Pont photopolymer and changed the limiting factor from the angular bandwidth of the optical system to the dynamic range of the material.

We thank Geoffrey Burr, Sidney Li, and Fai Mok for their helpful discussions.

Kevin Curtis is also with Northrop Corporation's B-2 Division, Pico Rivera, California 90660.

\section{References}

1. W. K. Smothers, T. J. Trout, A. M. Weber, and D. J. Mickish, presented at Second International Conference on Holographic Systems, Bath, UK, 1989.

2. K. Curtis and D. Psaltis, Appl. Opt. 31, 7425 (1992).

3. K. Curtis and D. Psaltis, in Annual Meeting, Vol. 23 of 1992 OSA Technical Digest Series (Optical Society of America, Washington, D.C., 1992), paper ThX3.

4. D. Psaltis, D. Brady, X. G. Gu, and S. Lin, Nature (London) 343, 325 (1990).

5. F. H. Mok, Opt. Lett. 18, 915 (1993).

6. H. Y. S. Li, "Photorefractive 3-D disks for optical data storage and artificial neural networks," Ph.D. dissertation (California Institute of Technology, Pasadena, Calif., 1994).

7. D. Brady and D. Psaltis, J. Opt. Soc. Am. A 9, 1167 (1992). 